[Vicino Oriente XVII (2013), pp. 153-164]

\title{
DEMETRA A MOZIA: \\ EVIDENZE DALL'AREA SACRA DEL KOTHON NEL V SECOLO A.C.
}

\author{
Federica Spagnoli - Sapienza Università di Roma
}

During the seasons of excavation 2010-2011 carried out by «La Sapienza» Expedition to Motya, several pits were identified near the southern corner of the Kothon. Two of them have been used for cult activities during the $5^{\text {th }}$ century $B C$, as attested to by botanical and faunal samples, votive offerings and sacred tools found inside them. Such evidence suggests that a chthonic cult devoted to a divine couple connected to underground water, to the fertility and to the cycles of nature was performed in the sacred area of the Kothon (Nigro 2012a). The main god has been identified with Baal 'Addir (Poseidon) and his companion can be now identified with Demetra, assimilated to the Phoenician goddess Astarte.

Keywords: Mozia; Astarte; Demetra; culto ctonio; sincretismo

\section{INTRODUZIONE}

Gli scavi della Missione archeologica a Mozia dell’Università di Roma «La Sapienza», condotti nel settore sud-occidentale dell'area sacra del Kothon tra il 2010 e il $2011^{1}$, hanno rivelato l'esistenza di sei pozzi posti a breve distanza gli uni dagli altri, scavati nel banco di marna argillosa regolarizzato al momento dell'insediamento della prima colonia fenicia ${ }^{2}$. Alcuni di questi pozzi ${ }^{3}$ furono in uso fino alla metà del VI secolo a.C. ${ }^{4}$, allorché l'area conobbe una risistemazione architettonica seguita alla distruzione che investì tutta l'antica città $^{5}$, due, invece, denominati P.2927 e P.1660, continuarono ad essere utilizzati ed hanno restituito evidenze archeologiche relative a un loro utilizzo cultuale almeno tra il 470 e il 397/6 a.C. ${ }^{6}$. L'analisi dei rinvenimenti effettuati in queste due installazioni sacre, sia quelli direttamente riferibili al rito che le offerte votive, offre alcuni spunti di riflessione sulla natura del culto che vi veniva officiato e sulla sua attribuzione.

1 Desidero ringraziare sentitamente il Professor Lorenzo Nigro, Direttore della Missione archeologica a Mozia, per la fiducia accordatami nell'affidarmi lo studio dei materiali illustrati nel presente contributo, per le indicazioni e i suoi preziosi suggerimenti. Un ringraziamento va anche ai colleghi Pierfrancesco Vecchio e Adriano Orsingher, responsabili di scavo dell'area C Sud-Ovest negli anni cui fa riferimento il presente lavoro. Infine, ringrazio il Professor Francesco Guizzi per i consigli e le puntuali osservazioni.

2 Nigro - Spagnoli 2012, passim; Nigro 2012b, 207-208; Nigro in questo volume

3 Si tratta dei pozzi denominati rispettivamente P.2351, P.2370, P.1673 e Well 3. Il pozzo P.2351 era stato già riconosciuto e denominato Well 1 dalla missione inglese diretta da B.S.J. Isserlin, insieme al Well 2 , localizzato più a sud. Il pozzo P.2351 rimase in uso anche nella Fase 5 (550-470 a.C.). Il Well 3, individuato più a sud, verso la costa, fu obliterato dalla costruzione della mura alla metà del IV secolo a.C.; un altro pozzo, denominato Well 4, è attribuibile al periodo successivo. Si veda da ultimo Nigro - Lisella 2004, 80-82, figg. 5, 9-10.

4 Nigro 2010c, 8-10.

$5 \quad$ Nigro 2013a, tab. 1

6 Tale range cronologico rappresenta la Fase 4 della periodizzazione generale dell'area del Kothon (Nigro [a cura di] 2005, 48-59). 


\section{I POZZI SACRI P.2927 E P.1660: STRATIGRAFIA E RINVENIMENTI}

I pozzi P.2927 e P.1660 sorgono a poche decine di metri a sud dell'angolo meridionale del Kothon e furono in uso già nelle prime fasi di occupazione fenicia dell'area nei periodi Mozia IV e $\mathrm{V}^{7}$. La stratigrafia del taglio, la quota dell'imboccatura dei pozzi, e il rinvenimento al loro interno di ceramiche ascrivibili ai Periodi IV e $\mathrm{V}^{8}$, testimoniano che furono scavati e utilizzati dalla fine dell'VIII secolo a.C. ${ }^{9}$.

Il pozzo P.2927 presenta anche nella Fase 4 un'imboccatura circolare costituita da piccole pietre calcaree irregolari; esso fu in utilizzato fino alla fine del $\mathrm{V}$ secolo a.C., quando fu chiuso ritualmente. Quest'ultima azione rituale è testimoniata dalle offerte relative al rito di obliterazione, rinvenute immediatamente sotto al tappo, costituito da terra argillosa mista a pietrame. Nello strato superiore del riempimento del pozzo erano un blocchetto a gola egizia e un versatoio ricavato dal collo di una lekythos associato a un fondo di skyphos, anch'esso ritagliato, utilizzato come vassoio ${ }^{10}$. Al di sotto erano le altre offerte: un'arula in terracotta spezzata, un frammento di louterion ${ }^{11}$, e vasi, principalmente coppe e skyphoi, a vernice nera e acromi, alcuni dei quali ritagliati per essere utilizzati nel rito $^{12}$. Le anfore rappresentano la classe ceramica più cospicua: sono state individuate numerose anfore greche e puniche; queste preservavano sulla superficie esterna il residuo del vino aromatizzato originariamente in esse contenuto, che venne poi versato ritualmente nel pozzo e utilizzato per la libagione rituale ${ }^{13}$. Tra le offerte erano anche una ventina di ciottoli sferici e oblunghi, a coppie uno bianco e uno nero ${ }^{14}$, un frammento di macina in basalto ${ }^{15}$, ossa animali, specialmente crani e stinchi ${ }^{16}$, una conchiglia, un astragalo, semi, mandorle e frammenti lignei ${ }^{17}$. Tutti gli oggetti deposti presentavano evidenti tracce di combustione ed erano frammisti a carboncini.

Il pozzo P.1660, localizzato circa un metro a est del precedente, già in uso almeno dalla fine dell'VIII secolo a.C. ${ }^{18}$, venne utilizzato, dopo la metà del VI secolo a.C., per scopi rituali, fino a che non fu chiuso e obliterato alla fine del V secolo a.C. L'imboccatura quadrata, con gli angoli orientati secondo i punti cardinali, è stata realizzata nel corso della

$7 \quad$ Nigro 2010c, 8, fig. 5; Nigro in questo volume

8 I Periodi IV e V della scansione cronologica generale di Mozia corrispondono alle Fasi 9-7 della periodizzazione dell'Area del Kothon, che coprono un arco cronologico di circa due secoli, dal 770 al 550 a.C. (Nigro 2013b).

9 Si veda nota 18

10 Rispettivamente MC.11.4514/2 e MC.11.4514/1 (Nigro - Spagnoli 2012, 38, figg. 6-7, 16-17, tav. I).

11 Il blocchetto di calcarenite lavorato a gola egizia (MC.11.RA.XX), l'arula (MC.11.117) e il louterion (MC.11.4514/3) potrebbero essere appartenuti a un sacello nelle vicinanze connesso al culto (Nigro - Spagnoli 2012, 35, figg. 5, 12, 15, tav. III).

12 Nigro - Spagnoli 2012, 40-41, tav. I

Nigro - Spagnoli 2012, 44-48, 50.

Ad esempio MC.11.108 e MC.11.109 (Nigro - Spagnoli 2012, fig. 8)

MC.11.118 (Nigro - Spagnoli 2012, fig. 9).

Alhaique 2012, 49, fig. 30

Nigro - Spagnoli 2012, 35, figg. 10-11

18 Nigro - Spagnoli 2012, 34-35, 51, figg. 3-4, 32-33. Nel pozzo P.1660 sono stati rinvenuti un frammento di kotyle corinzia (MC.11.4512/10: Nigro - Spagnoli 2012, 55, fig. 36), di ceramica punica dipinta (MC.11.4512/12, MC.11.4512/13) e di diverse pignatte e teglie d'impasto (MC.11.4512/23, MC.11.4512/24). 
Fase 5 (550-470 a.C.) utilizzando quattro blocchi in calcarenite ${ }^{19}$. Le offerte rituali rinvenute nel riempimento, a una profondità di circa 1 metro sotto al tappo di pietre e scheggioni calcarei, includevano una pietra pomice lavorata, un oscillum, un chiavistello di ferro, due astragali di bovino ${ }^{20}$, una falange di caprovino, tre frammenti di carapace e piastrone di testuggine ${ }^{21}$. La ceramica include due lucerne, una bolsal, il fondo ritagliato recante due lettere graffite (la dedica alla divinità) di una coppa a figure rosse ${ }^{22}$, e alcuni vasi da mensa, soprattutto skyphoi e fondi di coppe ritagliate ${ }^{23}$.

\section{AZIONI RITUALI E OFFERTE VOTIVE: I MODI E GLI OGGETTI DEL RITO}

Le offerte e gli oggetti deposti al momento della obliterazione dei pozzi P.2927 e P.1660 mostrano nei due contesti significative analogie (tab. 1): in entrambi i pozzi furono svolti rituali di libagione e di aspersione di acqua e vino ${ }^{24}$, testimoniati dalla presenza di coppie di bicchieri o skyphoi; il liquido veniva libato attraverso colli di vasi ritagliati e utilizzati come versatoi. In particolare, tale funzione era svolta da due colli di lekythoi appositamente tagliati e posti a guisa di mundus. Il rituale prevedeva anche la cottura di carne e cereali, come testimoniano i materiali carboniosi del riempimento, misti a frammenti di ceramica da cucina e da preparazione ${ }^{25}$. Gli animali impiegati erano principalmente suini e ovicaprini, i primi molto giovani ${ }^{26}$, i secondi di età differenti. L'atto sacrificale aveva luogo a breve distanza dai pozzi, probabilmente su una piattaforma ${ }^{27}$, laddove avveniva la macellazione e l'eviscerazione dell'animale. Le parti grasse e quelle meno nobili venivano offerte bruciandole, come testimoniano le deposizioni di parti craniali e di stinchi. Il rituale prevedeva, inoltre, l'offerta di semi e frutta secca non combusti, tradizionalmente posti entro coppette o su fondi di vasi ritagliati utilizzati come vassoi $^{28}$. Tra la ceramica greca e punica utilizzata per il culto $^{29}$, è possibile distinguere coppie di forme gemelle (bicchieri e brocche), accomunate dal tipo o dalle dimensioni e, evidentemente, dalla funzione rituale (libagione in coppia) ${ }^{30}$.

Gli oggetti offerti nel rituale di obliterazione sono vari: oggetti in pietra, in metallo e in ceramica, conchiglie, un carapace e piastrone di testuggine e ossa lavorate (astragali),

19 Nigro - Spagnoli 2012, 51, figg. 51-52.

20 Questi oggetti sono denominati rispettivamente MC.11.48, MC.11.82, MC.11.73, MC.11.87, MC.11.88 (Nigro - Spagnoli 2012, 51, tav. IV)

21 Nigro - Spagnoli 2012, 57, fig. 39

22 Come la coppa MC.11.4512/2 deposta nel pozzo P.1660 (Nigro - Spagnoli 2012, 53, fig. 35).

23 Nigro - Spagnoli 2012, 53-56, tav. V.

24 Come mostrano le numerose anfore vinarie rinvenute (Nigro - Spagnoli 2012, 44-48, tav. II).

25 Nigro - Spagnoli 2012, 41, tavv. I, III; 53-55, fig. 37, tav. IV.

26 I maialini non superano l'anno di età (Alhaique 2012, 49).

27 Nigro - Spagnoli 2012, 8.

28 Come il fondo ritagliato dello skyphos a figure rosse (MC.11.4514/1) rinvenuto in associazione al collo della lekythos in vernice rossa (MC.11.4514/2) nel pozzo P.2927 (Nigro - Spagnoli 2012, 38-40).

29 Nigro - Spagnoli 2012, 38-48, 53-56, tavv. I-III, V-VI.

30 Ad esempio una coppia di skyphoi dello stesso tipo nel pozzo P.2927 (MC.11.4514/18 e MC.11.4514/30) o la coppia di brocchette nel pozzo P.1660 (MC.11.4512/16 e MC.11.4512/20). Per casi analoghi in altri contesti cultuali dell'Italia meridionale si veda, a titolo esemplificativo, Fabbri - Osanna 2005, 220-221. 
bolsal e lucerne a vernice nera, e fondi di vasi figurati appositamente ritagliati per essere utilizzati come coppelle o vassoi (tab. 2).

Le offerte sono riconducibili ad un ambito cultuale di tipo ctonio: oggetti in pietra, la cui natura vulcanica (pomice e basalto) costituisce in sé un richiamo al mondo infero; oggetti di ferro, metallo anch'esso riferito alla sfera ctonia; semi, frutti della terra, conchiglie, che costituiscono il collegamento con il mondo marino. Il rito si svolgeva a contatto diretto con la terra e con l'acqua che affiorava dal pozzo, e prevedeva contemporaneamente l'uso di liquidi e del fuoco. Se, infatti, da un lato è attestata la libagione verso il mondo infero, effettuata tramite un mundus direttamente nel pozzo, che raggiungeva la falda delle acque sotterranee, dall'altro lato le tracce di bruciato riscontrate su molti degli oggetti e delle ossa rinvenute attestano l'esecuzione di una thysia, un tipo di pratica in cui l'offerta, sia che si tratti di un animale o di un vegetale, sia di un oggetto inanimato, viene bruciato o consumato per combustione.

Dall'analisi della ceramica non sembra che il pasto che seguiva il sacrificio animale ${ }^{31}$ venisse consumato nello stesso luogo dove questo si era svolto ${ }^{32}$. Non era presente, infatti, un numero di vasi da mensa e da fuoco tale da testimoniare un pasto collettivo e, tra la ceramica utilizzata per il rituale, non sono stati rinvenuti altri vasi se non quelli, a coppie, di cui si è detto in precedenza, collegabili più che altro alla libagione.

\begin{tabular}{|l|l|l|}
\hline AZIONI RITUALI & INDICATORI \\
\hline $\begin{array}{l}\text { LIBAGIONE/ASPERSIONE } \\
\text { DI VINO, ACQUA, ALTRO }\end{array}$ & P.2927 & $\begin{array}{l}\text { P.1660 } \\
\text { e vasi potori } \\
\text { Mortai } \\
\text { [Anfore vinarie e brocche] }\end{array}$ \\
\hline $\begin{array}{l}\text { COMBUSTIONE } \\
\text { DI INCENSI E PARTI ANIMALI } \\
\text { ESCHARA) }\end{array}$ & $\begin{array}{l}\text { Carboni } \\
\text { Vasi da fuoco }\end{array}$ & $\begin{array}{l}\text { Imbuti e vasi potori } \\
\text { Mortai } \\
\text { [Anfore vinarie e brocche] }\end{array}$ \\
\hline $\begin{array}{l}\text { OFFERTA ALIMENTARE } \\
\text { (TERRA/MARE) }\end{array}$ & $\begin{array}{l}\text { Vassoi ritagliati } \\
\text { Bue, maiali, ovicaprini } \\
\text { Molluschi }\end{array}$ & $\begin{array}{l}\text { Carboni } \\
\text { Vasi da fuoco }\end{array}$ \\
\hline OFFERTA DI SEMI & $\begin{array}{l}\text { Fondi ritagliati } \\
\text { Semi }\end{array}$ & $\begin{array}{l}\text { Vassoi ritagliati } \\
\text { Ovicaprini }\end{array}$ \\
\hline
\end{tabular}

Tab. 1 - Tabella sinottica con gli indicatori delle azioni rituali svolte nella cerimonia di obliterazione dei pozzi P. 2927 e P.1660.

31 Oltre alla carne, il pasto prevedeva probabilmente il consumo di cereali bolliti (Mastronuzzi 2008, 144-146).

32 La presenza di un fondo di un mortaio, o uno scodellone, ritagliato utilizzabile come vassoio (MC.11.4514/22) è legata probabilmente al rituale della presentazione dell'offerta che segue il sacrificio. Successivamente, le parti destinate alla divinità venivano depositate insieme agli altri oggetti, mentre il pasto veniva consumato altrove (Greco 2008, 45). Nei pasti rituali comuni, infatti, la prassi prevedeva che le stoviglie e gli utensili fossero lasciati nel tempio perché appartenenti anch'essi alla divinità, come attestato anche in contesti coevi analoghi sia della Sicilia che dell'Italia meridionale (Osanna - Giammatteo 2001, 109113; Osanna 2003, 261; Bailo Modesti et al. 2005, 47). 


\begin{tabular}{|c|c|c|}
\hline OFFERTE RITUALI & P.2927 & P.1660 \\
\hline CERAMICA & $\begin{array}{l}\text { Skyphos attico ritagliato (vassoio) } \\
\text { Coppie di vasi da mensa e preparazione } \\
\text { Lucerna }\end{array}$ & $\begin{array}{l}\text { Coppa a figure rosse ritagliata (vassoio) } \\
\text { Coppie di vasi da mensa e preparazione } \\
\text { Bolsal, lucerna }\end{array}$ \\
\hline OGGETTI IN PIETRA & Ciottoli (20) bianchi/neri & Pomice lavorata \\
\hline OGGETTI IN CERAMICA & - & Oscillum \\
\hline OGGETTI IN METALLO & - & Chiavistello di ferro \\
\hline $\begin{array}{l}\text { OSSA LAVORATE } \\
\text { E CONCHIGLIE }\end{array}$ & $\begin{array}{l}\text { Astragalo sx (bovino) } \\
\text { Astragalo sx (ovino) } \\
\text { Conchiglia (Glycimeris) }\end{array}$ & $\begin{array}{l}\text { Astragali } \\
\text { Carapace e piastrone di testuggine }\end{array}$ \\
\hline
\end{tabular}

Tab. 2 - Schema delle offerte votive dai pozzi P. 2927 e P.1660.

\section{UN CULTO DEDICATO A UNA COPPIA DIVINA}

L'analisi del contesto entro cui si svolse il rituale per l'obliterazione dei pozzi P.2927 e P.1660 suggerisce alcune indicazioni sulla natura della divinità, o delle divinità, cui il culto era tributato. La dualità del rito, testimoniata ad esempio nell'uso sia di acqua (libagione) sia di fuoco (thysia), può essere ricondotta a una coppia divina. La natura delle offerte rinvenute, inoltre, sembrerebbe richiamare la presenza di due opposti, rappresentati di coppie di ciottoli sferici bianchi e neri ${ }^{33}$, recuperati all'interno del riempimento del pozzo P.2927. Questa diade potrebbe essere costituita da una divinità maschile e una femminile. L'elemento maschile è da riconoscersi, con ogni probabilità, in Baal 'Addir/Poseidon ${ }^{34}$, come attestato da una iscrizione rinvenuta in prossimità dell'area dei pozzi ${ }^{35}$; il collegamento al mondo infero e alle acque sotterranee è rappresentato dagli oggetti in metallo e da quelli in pietra e dalle conchiglie. Il riferimento all'universo femminile può essere testimoniato dall'oscillum di terracotta, dagli astragali, dalla bolsal e dalle lucerne ${ }^{36}$.

Riguardo all'identificazione della divinità maschile con Baal 'Addir/Poseidon, va sottolineato come la presenza di conchiglie, ma soprattutto l'offerta del vino, per la quale si

33 Una suggestiva ipotesi circa l'interpretazione della presenza di ciottoli di colore diverso, chiaro e scuro o bianco e nero ci è suggerita ancora una volta dalle fonti greche: Pausania narra dell'esistenza a Figalia, presso il Monte Elaios in Arcadia, di un culto in grotta dedicato a Demetra Melaine (Nera), così chiamata per il colore della veste della statua lignea della dea: Pausania, VIII 42, 4 (Pausania, Guida della Grecia, Libro VIII, testo e traduzione a cura di M. Moggi, commento a cura di M. Moggi e M. Osanna, Milano 2003, 488 per il solo $§ 4$ (con rinvio a Sfameni Gasparro 1986, 326), 487-490 per tutto il cap.42).

34 Baal 'Addir, attestato a Biblo nel VI secolo a.C. con l'epiteto "Signore Potente", ricopre nel pantheon fenicio il ruolo di divinità ctonia e agraria. In epoca tarda, soprattutto in Nord Africa, è assimilato a Baal Hammon, il quale, proprio come Baal 'Addir, è riconducibile al Baal siriano, figlio di Dagan, signore della vita e della fertilità, della terra e dalla vegetazione, della tempesta e della pioggia, delle acque infere e delle acque marine (Lipińsky 1992, 55-56, 126).

35 Si tratta del frammento di aryballos laconico MC.10.2951/1 rinvenuto nella favissa F.2950 (vedi infra nota 47), che reca un'iscrizione dedicatoria in greco a Belos, il Baal fenicio (Guizzi 2012, 13-15, figg. 1-2; Nigro Spagnoli 2012, 32).

36 Il prevalente genere femminile dei destinatari di questo tipo di forme ceramiche, in particolare le lucerne, è testimoniato, nell'Età del Ferro, anche in contesti della madrepatria, ad esempio a Sarepta (Anderson 1988, passim). 
rimanda alla nota formula omerica ${ }^{37}$, ben si adattino alla figura divina titolare del tempio maggiore dell'area sacra $^{38}$, proprio per la loro afferenza all'ambito marino e alla navigazione ${ }^{39}$.

Il profilo che viene delineandosi per la figura divina femminile, invece, è compatibile con quello di Astarte, la paredra di Baal, cui era dedicato un tempietto a nord dell'edificio maggiore, nel suo aspetto ctonio, come divinità legata alla fecondità, alla rinascita e ai cicli vitali dell'uomo e della natura ${ }^{40}$. Esso, peraltro, sembra essere anche più direttamente riferibile, sia nei caratteri generali, sia nei rituali attestati dalle offerte (il sacrificio della testuggine, l'offerta di maialini), alla figura di Demetra. A un culto tipicamente demetriaco possono, infatti, essere ricondotti i semi rinvenuti nelle coppette e nei fondi ritagliati, la mandorla e le lucerne ${ }^{41}$. La testuggine è anch'essa un'offerta tradizionalmente associata alla dea, specie in ambito ionico ed etrusco ${ }^{42}$, sebbene non sia in realtà estraneo alla cultura fenicia, come testimoniano alcune rappresentazioni su sigilli rinvenuti a Tharros ${ }^{43}$. Uno di questi sigilli, oltre alla scena di sacrificio di testuggine, raffigura anche una grande conchiglia $^{44}$, elemento che richiama la simbologia associata a Poseidon ${ }^{45}$.

Nell'area sacra del Kothon, dunque, l'originaria coppia Baal 'Addir/Astarte, titolare dei templi maggiori del complesso sacro sin dall'VIII secolo a.C., in particolare nelle fasi più arcaiche $^{46}$, nel corso del V secolo a.C. ${ }^{47}$ viene progressivamente assimilata alla coppia

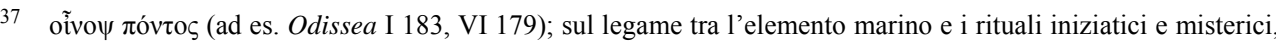
riconducibili principalmente alla sfera dionisiaca, D’Agostino 1999, 115-117.

38 Nigro 2010a, 22; Nigro 2012a, 294-301.

39 Bailo Modesti et al. 2005, 39

40 Sul culto di Astarte a Mozia e in generale sui culti femminili nella Sicilia Occidentale, si vedano Amadasi Guzzo 1981; Nigro 2010d; Spatafora 2010.

41 Torelli 1999, 685-686; Lippolis 2006, 137 segg.

42 Bonamici 2005, 5

43 Acquaro 1976, tav. XXIV; Acquaro 1984, 76-80, 84, fig. 101.

44 Si tratta di una Charonia Tritonis Nodifera, uno degli attributi di Tritone/Dagon, il figlio di Baal "Addir/Poseidon; un esemplare proviene dalla "Casa del corno di conchiglia" (Zona D) così chiamata proprio per questo particolare rinvenimento (Nigro 2012b, 210).

45 Nigro 2010b, 41-43.

46 Fasi 9-7, corrispondenti ai Periodi IV e V. Il culto della coppia divina Baal 'Addir/Astarte è attestato, oltre che nel Tempio C5, edificio maggiore dell'area sacra nei Periodi IV e V, anche in un'altra istallazione sacra, la favissa F.2950, posta a sud-ovest del Tempio, a ridosso dell'area occupata prima dall'Edificio C8 e poi dal Quartiere di Porta Sud. Nella Favissa F.2950 le offerte votive accumulate a seguito di azioni rituali reiterate nel corso di più di due secoli, e le testimonianze del rito effettuato in occasione della chiusura e della obliterazione della favissa, avvenuta alla metà del VI secolo a.C., attestano l'antichità del culto tributato a questa coppia nell'area sacra. Tra le offerte rituali, specialmente quelle relative all'utilizzo finale dell'installazione, numerosi sono i semeia riferibili a Baal 'Addir: oltre all'aryballos con iscrizione dedicatoria a Belos (vedi supra nota 35), altri elemento che riconducono a Baal 'Addir nel suo aspetto infero e legato alle acque marine, sono uno scarabeo in Egyptian Blue (Melandri 2012, 16-18, fig. 1), un rocchetto da rete (MC.10.262), un lisciatoio in pietra (MC.10.183) e, probabilmente, anche un'anfora in serpentite verde rinvenuta dagli archeologi inglesi nella stessa area. Anche il tipo di rituali attestati, cioè la libagione effettuata con il vino versato direttamente nell'acqua affiorante dal fondo della favissa attraverso un versatoio (costituito da un collo di anfora ritagliato, MC.10.2951/37) costituisce un ulteriore riferimento alla divinità maschile. Richiamano invece a un culto dedicato ad Astarte, come divinità di rinascita legata ai cicli della natura e della vita, lo svolgimento di una thysia, il pasto collettivo con carne, cereali e molluschi, di cui rimangono nella 
Poseidon/Demetra, pienamente inserita nella koiné siceliota dell'epoca ${ }^{48}$, specie per quanto riguarda la figura femminile, ma, in realtà, risalente ad antichissime tradizioni greche

Alcuni rinvenimenti effettuati in prossimità dei due pozzi confermano ulteriormente la sovrapposizione del culto di Demetra a quello di Astarte: una terracotta frammentaria in cui è rappresentata una mano (di divinità femminile in trono) che reca un melograno ${ }^{49}$, chiaro riferimento alla dea Malophoros ${ }^{50}$; la matrice fittile di una protome femminile con polos rinvenuta nel Sacello $C 7^{51}$, per la quale si richiama a un confronto con una protome proveniente da Selinunte databile agli ultimi decenni del VI secolo a.C. ${ }^{52}$. Dall'area sacra provengono, inoltre, numerose forme per focacce o per pani ${ }^{53}$ (i pani e i dolci della dea che si offrivano tradizionalmente) e delle testine femminili miniaturistiche ${ }^{54}$. Dagli scavi inglesi nella stessa regione dell'isola provengono oggetti che riconducono all'ambito demetriaco: una protome fittile femminile, delle coppette a vernice nera, alcune delle quali con lettere incise sul fondo, un piatto da pesce con il fondo appositamente forato ${ }^{55}$.

\section{CONCLUSIONI. IL CULTO DI DEMETRA A MOZIA: UN CASO DI INTERPRETATIO PUNICA?}

Nell'area sacra del Kothon il culto di Baal 'Addir e di Astarte è attestato fin dalle fasi più antiche: è presente nel Tempio $\mathrm{C} 5, \mathrm{C} 1$ e $\mathrm{C} 2^{56}$, e nel Tempietto di Astarte (Edificio C6, $\mathrm{C} 4)^{57}$. Nei rituali finora descritti, relativi all'obliterazione dei pozzi, le divinità appaiono adorate come coppia divina; in questa associazione sembra prevalere il carattere infero e ctonio di entrambe (l'uno legato alle acque sotterranee, l'altra alla terra) su tutte le altre

favissa tracce consistenti (ceramica da mensa e resti del pasto), i tipi e le età degli animali sacrificati (alcuni molto giovani, "primizie delle greggi") e la scelta del periodo autunnale per lo svolgimento del rituale (Nigro Spagnoli 2012, 8-11, 32).

47 Fasi 5-4, Periodi VI e VII (Nigro 2013a, tab. 1).

48 Ampolo 2012, 25 segg.

49 MC.08.210. Si tratta probabilmente della mano di una divinità femminile in trono. Per l'analisi stilistica e i confronti si rimanda allo studio sulle terrecotte figurate dagli scavi delle Campagne 2005-2008 nella Zona C di N. Chiarenza nel volume Mozia XIV di prossima pubblicazione.

50 "Colei che porta frutti" o "Colei che porta greggi": Pausania I 44, 3.

51 MC.13.100

52 Poma 2009, 246, n. 64. La matrice presenta alcuni elementi di derivazione ionica misti a tratti caratteristici della coroplastica siceliota, per cui si potrebbe avanzare l'ipotesi di un inquadramento cronologico entro la prima metà del V secolo a.C. Dalla Zona K proviene una protome con caratteristiche simili (Beer 2000, 12501251, fig. 4).

53 Per quelle meglio conservate (MC.09.65; MC.10.40; MC.11.30) si rimanda, come confronto, a Famà 2009, 270, nn. 14-16.

54 MC.08.233, MC.09.250

55 Isserlin 1971, tav. XXX:a-d. La testa femminile è riconducibile a una tipologia di ex voto molto frequente a Mozia e nei santuari magno-greci dedicati a Demetra e Kore dalla fine del VI secolo a.C. (Amadasi Guzzo 1969, 53-54, tavv. LXVIII-LXIV). Coppette e vasi incisi sul fondo (di solito una X, una A o una alef) o con il fondo appositamente forato caratterizzano i luoghi di culto ctonii (Torelli 1978, 214; Greco 1999, 244) e sono molto frequenti tra le offerte votive del Tempio C1-C2 e del Santuario C3 (Nigro 2009b, 704-705, figg. 7, 12).

56 Anche le installazioni cultuali che erano nell'Adyton del Tempio C5 lasciano presupporre lo svolgimento di riti di fuoco e di libagione (Nigro 2010c, 25-26, fig. 25; Spagnoli 2013, 90-91).

57 Nigro 2010a, 19-23. 
prerogative, specie quelle astrali ${ }^{58}$. La divinità femminile sarebbe, dunque, una interpretatio demetriaca $^{59}$ della figura di Astarte: alcune caratteristiche di questa dea fenicia poliedrica e ancestrale sarebbero trasferite tout court a Demetra ed elette a oggetto di un culto che attinge a ritualità e iconografie greche.

All'interno di questo quadro si delinea, per il settore sud-occidentale dell'area sacra, il ruolo di mediazione religiosa e culturale: qui è probabile che avessero luogo formule di ritualità ctonia frutto del sincretismo religioso tra tradizioni diverse, elime in primis e, successivamente, siceliote e greche. D'altra parte questo era uno dei punti nevralgici dell'area sacra, presso il quale affioravano stagionalmente le acque dolci ${ }^{60}$, posto all'ingresso meridionale dell'isola e immediatamente accessibile a chi entrasse nello Stagnone da sud ${ }^{61}$. Esso si configura quindi come luogo di culto interreligioso, un luogo vocato a culti legati ai cicli naturali e riproduttivi, particolarmente permeabile alla contaminazione e all'assimilazione di tradizioni religiose differenti, e a realtà altre rispetto all'universo religioso fenicio. Le conseguenze di questi fenomeni non si limitano alla sfera religiosa, ma comportano anche implicazioni culturali e sociali: la cultualità ctonia, praticata esclusivamente dalle donne ${ }^{62}$, rappresenta, seppur nella modalità descritta dalla formula "cittadinanza latente" (una cittadinanza, cioè, di tipo cultuale) ${ }^{63}$, un valido veicolo di mediazione e integrazione delle componenti etniche indigene, siceliote e greco-coloniali che diventeranno, con un processo di graduale assimilazione e contaminazione in atto sin dalla fine dell'VIII secolo a.C. (Mozia V), gli elementi caratterizzanti della cultura moziese del V secolo a.C.

58 Il carattere astrale del culto di Baal e della sua paredra, officiato nel Tempio del Kothon, trova riscontro nel tipo di oggetti che erano nel tempio: davanti alla base di stele nella Navata Orientale, ad esempio, è stato rinvenuto, infisso nel pavimento, il puntatore di uno strumento per misurazioni astrali, forse un astrolabio, e i numerosi fori nello stesso pavimento indicano che ve ne dovevano essere degli altri. L'orientamento stesso della fabbrica sacra e la posizione degli arredi sacri (betili e stele) confermano come la componente astrale fosse alla base della concezione del culto stesso, tanto da influenzare l'orientamento della fabbrica sacra: le due triadi betiliche presenti nel tempio, una nella Navata Orientale e l'altra nella Corte Centrale, sono orientate secondo il sorgere della costellazione di Orione rispettivamente nell'equinozio di primavera (a $\left.200^{\circ}\right)$ e nel solstizio d'inverno (a $\left.110^{\circ}\right)$. I due allineamenti di betili, le triadi, appunto, corrispondono agli assi minore e maggiore dell'edificio, che quindi sembra essere stato concepito ab origine secondo tale orientamento (Nigro 2009a, 262-265; Nigro 2010a, 19-22, figg. 8-10; Nigro 2010d, 163).

59 Questo fenomeno non rappresenta certamente una novità nel panorama mediterraneo: un processo di assimilazione analogo avviene in ambito etrusco, laddove Vei, divinità con prerogative astrali e ctonie legate alla "forza vitale" e al mondo infero, assume, dal VI secolo a.C., le caratteristiche di Demetra/Cerere (Belelli 2012, 462-464)

60 Sulla valenza e la funzione dei santuari periferici o liminari si vedano: Veronese 2006, 56; Tantillo 2012 passim.

61 I luoghi di culto delle divinità ctonie sorgono di solito vicino al mare o in corrispondenza di corsi d'acqua (Veronese 2006, 542; Tantillo 2012, 641).

62 Sfameni Gasparro 1986, 195 segg

63 Detienne - Vernant 1979, 187 


\section{BIBLIOGRAFIA}

ACQUARO, E.

1976 Componenti etrusco-ioniche nella glittica tharrense: Rivista di Studi Fenici IV/2 (1976), pp. 167-170.

1984 Arte e cultura punica in Sardegna (Sardegna Archeologica, Studi e Monumenti 2), Sassari 1984.

Alhaique, F.

2012 Resti faunistici dal pozzo P.2927: L. NigRo - F. SPAGnoli, Alle sorgenti del Kothon. Il rito a Mozia nell'Area sacra di Baal 'Addir - Poseidon. Lo scavo dei pozzi sacri nel Settore C Sud-Ovest (2006-2011) (Quaderni di Archeologia Fenicio-Punica/CM 02), Roma 2012, p. 49.

AMADASI GuZZO, M.G.

1969 Catalogo delle terrecotte: A. CIASCA ET ALII, Mozia - V. Rapporto preliminare della Missione congiunta con la Soprintendenza alle Antichità della Sicilia Occidentale (Studi Semitici 31), Roma 1969, pp. 53-104.

1981 Culti femminili a Mozia: Rivista di Studi Fenici 9 (1981), Supplementum, pp. 7-11.

AMPOLO, C.

2012 Compresenza di ethne e culture diverse nella Sicilia Occidentale. Per una nuova prospettiva storica: F. BERLINZANI (a cura di), Convivenza etniche, scontri e contatti di culture in Sicilia e Magna Grecia (Aristonothos - Scritti per il Mediterraneo antico, NIC 07), Trento 2012, pp. 15-57.

ANDERSON, W.P.

1988 Sarepta I. The Late Bronze Age and the Iron Age Strata of Area II, Y (Publications de l’Université Libanaise, section des Études Archéologiques, II), Beyrouth 1988.

BAILO MODESTI ET ALII

2005 Le acque intorno agli dei: rituali e offerte votive nel santuario settentrionale di Pontecagnano: M. BONGHI JOVINO - F. CHIESA (a cura di), Offerte dal regno vegetale e dal regno animale nelle manifestazioni del sacro, Atti dell'Incontro di Studio. Milano 26-27 giugno 2003 (Tarchna Supplementum 1), Roma 2005, pp. 37-63.

BEER, C.

2000 Two female terracotta protomai from Motya, Area K (campaign 1991): M.E. AuBET - M. BARTHÉlemy (eds.), Actas del IV Congreso Internacional de Estudios Fenicios y Púnicos, BELELLI, V. Cádiz, 2 al 6 de Octubre de 1995, Cádiz 2000, pp. 1249-1254.

2012 Vei: nome, competenze e particolarità cultuali di una divinità etrusca: V. Nızzo - L. LA RoccA (a cura di), Antropologia e archeologia a confronto: rappresentazioni e pratiche del sacro. Atti del secondo Incontro Internazionale di studi, Roma, Museo Nazionale Preistorico Etnografico “Luigi Pigorini”, 20-21 maggio 2011 (Antropologia e archeologia a confronto 2), Roma 2012, pp. 455-475.

BONAMICI, M.

2005 Appunti sulle pratiche cultuali nel santuario dell'acropoli volterrana: M. BONGHI JOVINO F. CHIESA (a cura di), Offerte dal regno vegetale e dal regno animale nelle manifestazioni del sacro, Atti dell'Incontro di Studio. Milano 26-27 giugno 2003 (Tarchna Supplementum 1), Roma 2005, pp. 1-10.

D’Agostino, B.

1999 Oinops pontos. Il mare come alterità nella percezione arcaica: B. D'AGostino - L. CERCHIAI (a cura di), Il mare, la morte, l'amore. Gli Etruschi, i Greci e l'immagine 
(Mélanges de l'École française de Rome. Antiquité T. 111, n. 1), Roma 1999, pp. 107117.

DETIENNE, M. - VERNANT, J.-P.

1979 La cuisine du sacrifice en pays grec (Bibliothèques des Histoires), Paris 1979.

DE VINCENZO, S.

2012 Tra Cartagine e Roma: I centri urbani dell'eparchia punica di Sicilia tra VI e I secolo a.C. (Topoi. Berlin Studies of the Ancient World), Berlin 2012.

FABBRI, M. - OSANNA, M.

2005 Aspetti del sacro nel mondo apulo: rituali di abbandono tra area sacrae abitato nell'antica Ausculum: M.L. NAVA - M. Osanna (a cura di), Lo spazio del rito. Santuari e culti in Italia meridionale tra Indigeni e Greci, Atti delle giornate di Studio (Matera, 28-29 giugno 2002) (Siris. Studi e ricerche della scuola di specializzazione in Archeologia di Matera, Supplementum 1), Bari 2005, pp. 215-233. FAMÀ, M.L.

2009 Arule, oggetti di uso domestico e oscilla figurati: M.L. FAMÀ (a cura di), Il Museo GRECO, G. Regionale "A. Pepoli” di Trapani. Le collezioni archeologiche, Bari 2009, pp. 257-275.

1999 Santuari extraurbani tra periferia cittadina e periferia indigena: AA.VV., La colonisation greque en Méditerranée Occidentale. Actes de la rencontre scientifique en hommage à Georges Vallet organiseé par le Centre Jean-Bérard, l'Ecole Française de Rome, l'Istituto Universitario Orientale et l'Università degli Studi di Napoli «Federico II», Rome-Naples, 15-18 novembre 1995 (Collection de l'Ecole Française de Rome 251), Rome 1999, pp. 231-247.

2008 Strutture per un sacrificio: G. GRECO - B. FERRARA (a cura di), Doni agli dei. Il sistema dei doni votivi nei santuari, Atti del Seminario di Studi (Napoli 21 aprile 2006), Pozzuoli 2008, pp. 29-48.

GuIZZI, F.

2012 Graffito con dedica votiva su un aryballos dall'area sacra del Kothon a Mozia: L. NigRO F. Spagnoli, Alle sorgenti del Kothon. Il rito a Mozia nell'Area sacra di Baal 'Addir Poseidon. Lo scavo dei pozzi sacri nel Settore C Sud-Ovest (2006-2011) (Quaderni di ISSERLIN, B.S.J. Archeologia Fenicio-Punica/CM 02), Roma 2012, pp. 13-15.

1971 New light on the "cothon" at Motya: Antiquity XLV (1971), pp. 178-186.

LIPINSKY, E.

1992 Dictionnaire de la Civilisation Phénicienne et Punique, Paris 1992.

LIPPOLIS, E.

$2006 \quad$ Mysteria: archeologia e culto del santuario di Demetra a Eleusi, Milano 2006.

MASTRONUZZI, G.

2008 Il culto di Demetra in Messapia: C.A. Di STEFAno (a cura di), Demetra. La divinità, $i$ santuari, il culto, la leggenda, Atti del I Convegno Internazionale (Enna, 1-4 luglio 2004), Pisa - Roma 2008, pp. 137-153.

MELANDRI, I.

2012 Uno scarabeo in Egyptian Blue dalla favissa F.2950: L. Nigro - F. Spagnoli, Alle sorgenti del Kothon. Il rito a Mozia nell'Area sacra di Baal 'Addir - Poseidon. Lo scavo dei pozzi sacri nel Settore C Sud-Ovest (2006-2011) (Quaderni di Archeologia FenicioPunica/CM 02), Roma 2012, pp. 16-19.

NigRo, L.

2009a Il Tempio del Kothon e il ruolo delle aree sacre nello sviluppo urbano di Mozia dall'VIII al IV secolo a.C.: S. Helas - D. MARzoli (Hrsg.), Phönizisch und punisches Städtewesen. 
Akten der internationalen Tagung in Rom vom 21. bis 23. Februar 2007 (Iberia Archaeologica Band 13), Mainz am Rhein 2009, pp. 241-270.

2009b Offerte e depositi votivi nel Santuario C3 del Kothon di Mozia nel IV secolo a.C.: S. ForTUNELli - C. MASSERIA (a cura di), Ceramica attica da santuari della Grecia, della Ionia e dell'Italia (Atti Convegno Internazionale Perugia 14-17 marzo 2007), Venosa 2009, pp. 703-719.

2010a L'orientamento astrale del Tempio del Kothon di Mozia: E. AnTONELLo (ed.), Il cielo e l'uomo: problemi e metodi di astronomia culturale. Atti del VII Convegno Nazionale della Società Italiana di Archeoastronomia. Roma, Museo Nazionale Romano, Terme di Diocleziano. 28-29 settembre 2007, Roma 2010, pp. 15-24.

2010b Il corno di Tritone: Archeo 306 (Agosto 2010), pp. 38-43.

2010c Alle origini di Mozia: stratigrafia e ceramica del Tempio del Kothon dall'VIII al VI secolo a.C.: L. Nigro (a cura di), Motya and the Phoenician Ceramic Repertoire between the Levant and the West, $9^{\text {th }}-6^{\text {th }}$ Century BC. Proceedings of the International Conference held in Rome, $26^{\text {th }}$ February 2010 (Quaderni di Archeologia Fenicio-Punica V), Roma 2010, pp. 1-48.

2010d Il Sacello di Astarte e i culti femminili a Mozia: G. BARTOLOnI - P. MatthiAe - L. Nigro L. Romano (a cura di), Tiro, Cartagine, Lixus: nuove acquisizioni. Atti del Convegno Internazionale in onore di Maria Giulia Amadasi Guzzo, Roma 24-25 novembre 2008 (Quaderni di Vicino Oriente IV), Roma 2010, pp. 163-180.

2012a The Temple of the Kothon at Motya, Sicily: Phoenician Religious Architecture from the Levant to the West: M. Gruber ET ALII (eds.), All the Wisdom of the East. Studies in Near Eastern Archaeology and History in Honor of Eliezer D. Oren (Orbis Biblicus et Orientalis 255), Friburg 2012, pp. 293-331.

2012b Scavi e restauri dell'Università di Roma 'La Sapienza' a Mozia, 2007-2009: il Tempio del Kothon, il Temenos Circolare, il Sacello di Astarte e il Tofet: C. Ampolo (a cura di), Sicilia occidentale. Studi, rassegne, ricerche. Atti delle settime giornate internazionali di studi sull'area elima e la Sicilia occidentale nel contesto mediterraneo, Erice, 12-15 ottobre 2009. Workshop «G. Nenci» diretto da Carmine Ampolo, Vol. II (Seminari e Convegni 29), Pisa 2012, pp. 207-218.

2013a Il Tofet e la città. Il limite meridionale del Santuario e le strutture collegate negli scavi della Sapienza 2010-2011: Scienze dell'Antichità 19/1 (2013), pp. 35-51.

2013b Before the Greeks: the earliest Phoenician settlement in Motya - recent discoveries by Rome «La Sapienza» Expedition: Vicino Oriente XVII (2013), pp. 39-74.

NigRo, L. (a cura di)

2005 Mozia - XI. Il Tempio del Kothon. Rapporto preliminare delle campagne di scavi XXIII e XXIV (2003-2004) condotte congiuntamente con il Servizio Beni Archeologici della Soprintendenza Regionale per i Beni Culturali e Ambientali di Trapani (Quaderni di Archeologia Fenicio-Punica II), Roma 2005.

NigRo, L. - Lisella, A.R.

2004 Il Quartiere di Porta Sud: L. NigRo - G. Rossoni (a cura di), «La Sapienza»a Mozia. Quarant'anni di ricerca archeologica, 1964-2004. Catalogo della mostra, Università di Roma «La Sapienza», Facoltà di Scienze Umanistiche, Museo dell'Arte Classica, 27 febbraio - 18 maggio 2004, Roma 2004, pp. 78-83.

Nigro, L. - Spagnoli, F.

2012 Alle sorgenti del Kothon. Il rito a Mozia nell'Area sacra di Baal 'Addir - Poseidon. Lo scavo dei pozzi sacri nel Settore C Sud-Ovest (2006-2011) (Quaderni di Archeologia Fenicio-Punica/Colour Monograph 02), Roma 2012. 
OSANNA, M.

2003 L'insediamento indigeno di Torre di Satriano (PZ): le nuove ricerche dell'Università degli Studi della Basilicata: Siris 3 (2003), pp. 233-268.

OSANNA, M. - GiAMMATTEO, T.

2001 Azioni rituali e offerte votive: M.L. NAVA - M. OsANNA (a cura di), Rituali per una dea Lucana: il Santuario di Torre di Satriano. Catalogo della Mostra (Soprintendenza Archeologica della Basilicata, Consiglio Regionale di Basilicata), Potenza 2001, pp. 107 122.

POMA, L.

2009 Le terrecotte figurate arcaiche e classiche: M.L. FAmÀ (a cura di), Il Museo Regionale "A. Pepoli” di Trapani. Le collezioni archeologiche, Bari 2009, pp. 223-248.

SFAMENI GASPARRO, G.

$1986 \quad$ Misteri e culti mistici di Demetra, Roma 1986.

SPAGNOLI, F.

2013 Phoenician cities and water: the role of the sacred sources in to the urban development of Motya, Western Sicily: T. TVedt - T. OestigaArd (eds.), A History of Water, Series 3, Vol. 1. From Jericho to Cities in the Seas: A History of Urbanization and Water Systems, London 2013, pp. 86-103.

SPATAFORA, F.

2010 Attestazioni di culti femminili nei santuari della Sicilia Occidentle: E. ACQUARO - A. FILIPPI - S. MEDAs (a cura di), La devozione dei naviganti. Il culto di afrodite ericina nel Mediterraneo. Atti del convegno di Erice, 27-28 novembre 2009 (Biblioteca di Byrsa 7), Lugano 2010, pp. 137-152.

TANTILLO, I.

2012 Alcune riflessioni sull'organizzazione spaziale dei santuari demetriaci periferici delle poleis siceliote in Età Arcaica: V. Nizzo - L. LA RoccA (a cura di), Antropologia e archeologia a confronto: rappresentazioni e pratiche del sacro. Atti del secondo Incontro Internazionale di studi, Roma, Museo Nazionale Preistorico Etnografico "Luigi Pigorini”, 20-21 maggio 2011 (Antropologia e archeologia a confronto 2), Roma 2012, pp. 641-652.

TORELLI, M.

1978 La ceramica ionica in Etruria: il caso di Gravisca: AA.VV., Les Cèramiques de la Gréce de l'Est et leur diffusion en Occident. Centre Jean Bérard. Institut français de Naples, 6-9 juillet 1976 (Colloques Internationaux du Centre National de la Recherche Scientifique $\mathrm{n}$. 569, Sciences Humaines), Paris - Naples 1978, pp. 213-215.

1999 Santuari, offerte e sacrifici nella Magna Grecia della frontiera: G. PUGLIESE CARRATELLI M. BAts (a cura di), Confini e frontiera nella Grecia d'Occidente. Atti del trentesimo convegno di studi sulla Magna Grecia, Taranto 3-6 ottobre 1997, Napoli 1999, pp. 685705 .

VERONESE, F.

2006 Lo spazio e la dimensione del sacro. Santuari greci e territorio nella Sicilia arcaica (Saggi di antichità e tradizione classica 24, nuova serie), Padova 2006. 